\title{
PRIMER REGISTRO FÓSIL DE UN MYRIAPODA (POLYDESMIDA) PARA EL PLEISTOCENO TARDÍO DE LA LOCALIDAD DE LA PALMERA DE SAN CARLOS, PROVINCIA DE ALAJUELA, COSTA RICA
}

\author{
THE FIRST FOSSIL RECORD OF A MYRIAPODA (POLYDESMIDA) FROM \\ THE LATE PLEISTOCENE LOCALITY OF LA PALMERA DE SAN CARLOS, \\ ALAJUELA PROVINCE, COSTA RICA
}

\author{
César A. Laurito ${ }^{1,2 *}$ y Ana L. Valerio ${ }^{3}$ \\ ${ }^{1}$ INA, Instituto Nacional de Aprendizaje \\ ${ }^{2}$ Investigador Asociado-Departamento de Historia Natural, \\ Museo Nacional de Costa Rica \\ ${ }^{3}$ Departamento de Historia Natural, Museo Nacional de Costa Rica. \\ Apdo. 749-1000, San José \\ *Autor para contacto: cesarlaurito@ice.co.cr
}

(Recibido: 28/08/2017; aceptado: 31/10/2017)

\begin{abstract}
For the first time is recorded a millipede (Diplopoda, Polydesmida) fossil material from Costa Rica and Central America; this material was recovered from travertine sedimentary rocks and was associated to osseous remains of several species of fossil mice as well as abundant flora fossil from the late Pleistocene. The fossil remains are basically disarticulated trunk segments and are closely related to present-day genus Nyssodesmus Cook, 1896. Keywords: Diplopoda, Polydesmida, Platyrhacidae, Late Pleistocene, Costa Rica.

RESUMEN: Se describe por primera vez para Costa Rica y América Central un milpiés (Diplopoda, Polydesmida) fósil; el material fue recolectado de depósitos de travertino y estaban asociados a varios restos óseos de roedores fósiles así como abundante flora fósil del Pleistoceno tardío. Los restos fósiles están básicamente constituidos por segmentos corporales y están cercanamente emparentados al género viviente Nyssodesmus Cook, 1896.

Palabras clave: Diplopoda, Polydesmida, Platyrhacidae, Pleistoceno Tardío, Costa Rica.
\end{abstract}

Laurito, C. A. y Valerio, A. L. (2018). Primer registro fósil de un Myriapoda (Polydesmida) para el Pleistoceno Tardío de la localidad de la Palmera de San Carlos, provincia de Alajuela, Costa Rica. Revista Geológica de América Central, 58, 179-187. doi: 10.15517/rgac.v58i0.32848 


\section{INTRODUCCIÓN}

La Clase Diplopoda incluye los artrópodos conocidos coloquialmente como milpiés, y constituyen un grupo terrestre muy antiguo, altamente diverso con más de 12000 especies nominales y una diversidad planetaria de poco más de 80000 especies, distribuidas en todos los continentes a excepción de la Antártida (Hoffman et al., 2002). El tronco segmentado está compuesto de un número de anillos que le dan una apariencia cilíndrica, raramente aplanada. Cada anillo está formado por dos segmentos o diplosegmentos que se fusionan posteriormente al cuarto segmento y tienen 2 pares de patas. El tegumento está muy calcificado y presentan glándulas odoríferas características (cf. Melic y Grustán, 1996; Shear y Edgecombe, 2010). Hoffman (1969) hizo la observación de que ninguno de los órdenes sobrevivientes de miriápodos actuales, presentan registros fósiles anteriores al Oligoceno y Shear (1981) considera que ello se debe a que este grupo es muy antiguo y como ocurre con todos los artrópodos, se caracteriza por una lenta evolución, por lo que no es de extrañar que las formas del Oligoceno o posteriores sean similares a las actuales.

Los miriápodos fósiles son extraordinariamente raros y escasos, porque son artrópodos continentales, subaéreos que suelen vivir en hábitats que no favorecen la preservación de sus exoesqueletos, de por sí frágiles y fácilmente degradables, no obstante, su relativo alto grado de calcificación, de allí que raramente se encuentren ejemplares completos y articulados (Melic y Grustán, 1996).

Se desconoce cuál es el grupo basal de los Myriapoda, pero la evidencia fósil sugiere que dicho grupo tuvo que surgir en el Cámbrico temprano, con un periodo cladogenético importante que abarcó desde el Ordovícico tardío al Silúrico temprano, conquistando la tierra firme en el Silúrico medio (Shear y Edgecombe, 2010).
Wilson et al. (2004), dan a conocer el registro certero de Myriapoda, procedentes del Silúrico temprano de Milwaukee en Estados Unidos. Los Diplopoda propiamente dichos, se presume aparecieron en el Devónico medio de acuerdo con el registro de fósiles en la localidad de Cowie Harbour, Escocia (Almond, 1985; Wilson y Anderson, 2004; Shear y Edgecombe, 2010).

El periodo Carbonífero cuenta con el registro Paleozoico más diverso de Myriapoda algunos de ellos pudieron tener hábitos anfibios como los arquidiplópodos (Hoffman, 1969), este periodo geológico, tiene un registro sesgado hacia los Diplopoda, asociados por lo general a hábitats muy restringidos; por el contrario, el periodo Pérmico cuenta con muy pocos registros. De igual forma el Mesozoico, se caracteriza en general, con escasos registros aislados (Shear et al., 2009); el Cenozoico, también se caracteriza por unos pocos registros de Myriapoda, preservados en ámbar y asignables principalmente a taxones vivientes (Hoffman, 1969; SantiagoBlay y Poinar, 1992; Shear y Edgecombe, 2010; Riquelme et al., 2014a y b).

En el Caribe, aunque raros, no son infrecuentes los registros fósiles de Myriapoda y usualmente provienen de ambientes cársticos o son ejemplares encapsulados en ámbar, asociados a climas tropicales húmedos y rocas carbonatadas, registrándose 3 géneros y especies de Diplopoda, a saber Rhinocricus sp., Chondrotropis sp. y Caraibodesmus verrucosus (Pockov) en el Pleistoceno Superior u Holoceno de Jamaica (Donovan y Veltkamp, 1994). Para el Oligoceno Superior de la Cordillera Septentrional en la República Dominicana, se han registrado 10 familias y 11 géneros fósiles de Myriapoda preservados en ámbar, todos con representantes actuales (Shear, 1981; Santiago-Blay y Poinar, 1992; Pérez-Asso y Pérez-Gelabert, 2001). Para la región de Mesoamérica, específicamente para el Mioceno del Municipio de Simojovel de Allende en el Estado de Chiapas, México, 


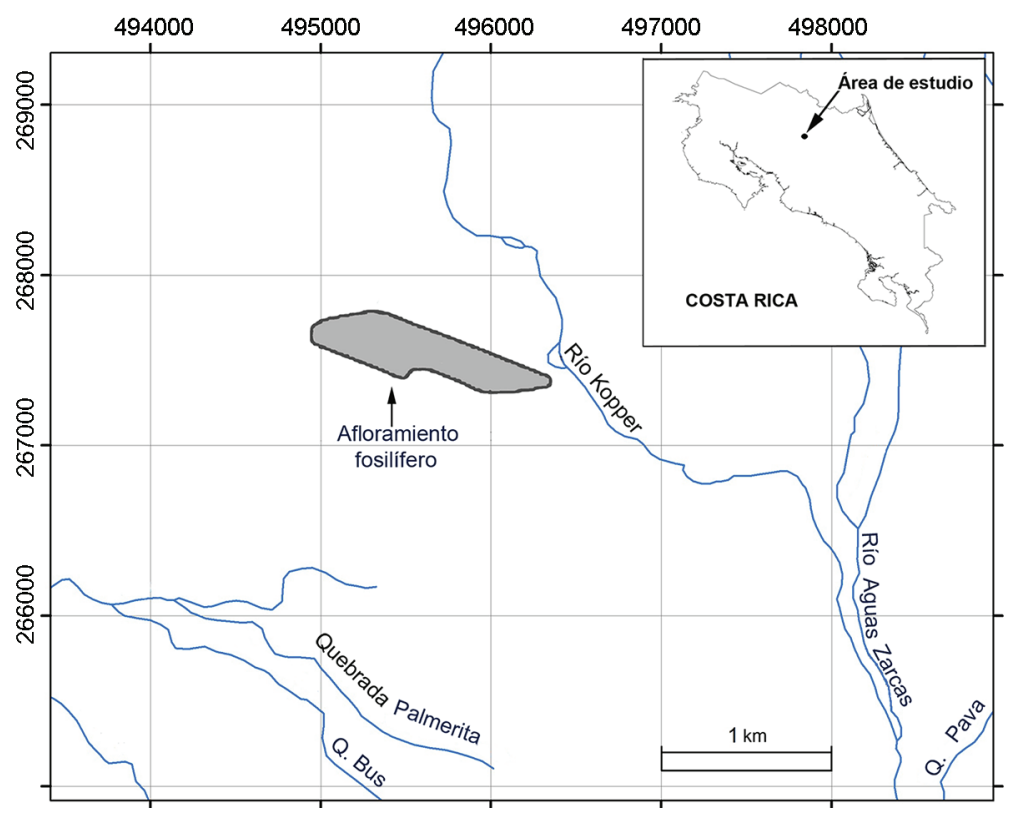

Fig. 1: Mapa de ubicación de la localidad fosilífera de La Palmera de San Carlos en la provincia de Alajuela.

Riquelme et al. (2014a y b), describen los géneros y especies fósiles Parastemmiulus electron, Maatidesmus paachtun y Anbarrhacus adamantis todos preservados en ámbar.

En Costa Rica, Pérez (2001) y Pérez y Laurito (2003) mencionan por primera vez la presencia de diplópodos fósiles en la localidad de La Palmera de San Carlos (ver figura 1), asociados a un importante yacimiento paleobotánico del Pleistoceno Superior. Éste material fósil se encuentra depositado en la colección de la Sección de Geología del Museo Nacional de Costa Rica y es el objeto de estudio del presente informe.

\section{GEOLOGÍA}

El afloramiento de travertino (sensu Pérez, 2001; Pérez y Laurito, 2003) se asocia a un sistema de fallas escalonadas de rumbo NW-SE, paralelo a la margen norte del volcán Platanar, por el cual emergen aguas termales enriquecidas en carbonato de calcio, sales que se asume provienen de los arrecifes del Mioceno Medio de la Formación Venado que afloran en la región. El afloramiento cuenta con una longitud de $1300 \mathrm{~m}$ de largo y $200 \mathrm{~m}$ de ancho y un espesor visible de $40 \mathrm{~m}$ y se presume sobreyace depósitos piroclásticos diversos. Los bancos de travertino son sobreyacidos por capas de ceniza y en algunos puntos se interdigitan con capas de toba.

\section{PALEONTOLOGÍA}

La taxonomía que a continuación se presenta, se basa en la revisión de la clasificación de los Diplopoda a nivel de familias, realizada por Shelley (2002). 
Clase Diplopoda de Blainville en Gervais, 1844 Infraclase Helminthomorpha Pocock, 1887

Superorden Merocheta Cook, 1895

Orden Polydesmida Pocock, 1887

Suborden Leptodesmidea Brölemann, 1916

Superfamilia Platyrhacoidea Pocock, 1895

Familia Platyrhacidae Pocock 1835

Género Nyssodesmus Cook, 1896

Nyssodesmus sp. Indet.

Material: el ejemplar CFM-5199, aglomerado en travertino constituido por aproximadamente 180 diplosomitos, la mayoría se encuentran desarticulados y recubiertos por una capa importante de carbonato de calcio, pero en unos cuantos casos es posible observar varios diplosegmentos articulados.

Descripción: diplosegmentos de contorno circular; metazonita con paranota cortos y dispuestos horizontalmente, las superficies del metatergito y los paranota, parecen ser lizas y carentes de escultura, donde están visibles (Fig. 4C-D). Los paranota con contorno anterior ligeramente cóncavo y contorno posterior convexo con espinas terminales cortas, ligeramente infladas con extremos redondeados.

Contacto entre la prozonita y el metazonita constituida por una línea de sutura apenas visible y una cintura muy angosta (Fig. 4C-D).

Diplosternitas típicas con 4 agujeros, ligermente más anchas que largas, protuberantes cuyo contorno recuerda a la silueta de un reloj de arena.

\section{Paleoecología y edad}

El yacimiento de La Palmera de San Carlos, se ubica a 400 m.s.n.m., su asociación paleoflorística se caracteriza por la presencia del roble Quercus corrugata Hooker (Pérez y Laurito, 2003) y el nogal Juglans olanchana Standley y L.O. Williams (Pérez, 2003), flora que en la actualidad es posible ubicarla a más de 1200 y hasta los 2000 m.s.n.m. para el caso del roble (Burger, 1977) y los 400 a los 1100 m.s.n.m. para el caso del nogal (Standley y Williams, 1950). Además, debe tenerse en cuenta que el género Juglans en la actualidad se distribuye desde el norte de los Estados Unidos hasta Nicaragua y desde Colombia y Venezuela hasta Argentina (Stone, 1977), por lo que la ausencia o extirpación de dicho género en Costa Rica y Panamá, sólo se explica por un cambio climático abrupto que provocó una migración latitudinal y altitudinal de los nogales. Por lo que se considera que la presencia de los géneros Juglans y Quercus en dicho yacimiento se asume fue concomitante o posterior al Pleniglacial del Pleistoceno Superior (Pérez y Laurito, 2003; Pérez, 2003).

Por otra parte, fueron extraídos de la muestra CFM-5199 cuatro especies de roedores fosilizados que cuentan con representación actual: Tylomys watsoni, Reithrodontomys mexicanus, Sigmodon hispidus y Proechimys semispinosus (Laurito, 2003).

Esta asociación de micromamíferos refuerza la paleoecología que previamente se tenía de la localidad de La Palmera de San Carlos, que a pesar de hallarse a solo 400 m.s.n.m., coincide con la zona de vida actual Bosque Premontano transición al Bosque Montano Bajo, equivalente al dominio altitudinal de 1200 m.s.n.m. a los 1300 m.s.n.m. Ello permite concluir un ambiente mucho más frío, lo que a su vez sugiere una edad probable Pleistoceno Superior para el yacimiento de La Palmera (Laurito, 2003).

Por su parte los milpiés, se sabe forman parte de la fauna del suelo y son considerados los macrodetritívoros terrestres más antiguos, especializados en la degradación de la materia orgánica de origen vegetal. De igual forma, representan una fracción importante de la biomasa total de detritívoros en muchos ecosistemas templados y tropicales (Crawford, 1992).

\section{DISCUSIÓN Y CONCLUSIONES}

La presencia en la región ventral de los diplosomitos con esternitas pares, evidencia la inserción de dos pares de apéndices segmentados o piernas, ello permite identificar de manera confiable que los restos pertenecen a la Clase Diplopoda (Fig. 3A-B). 


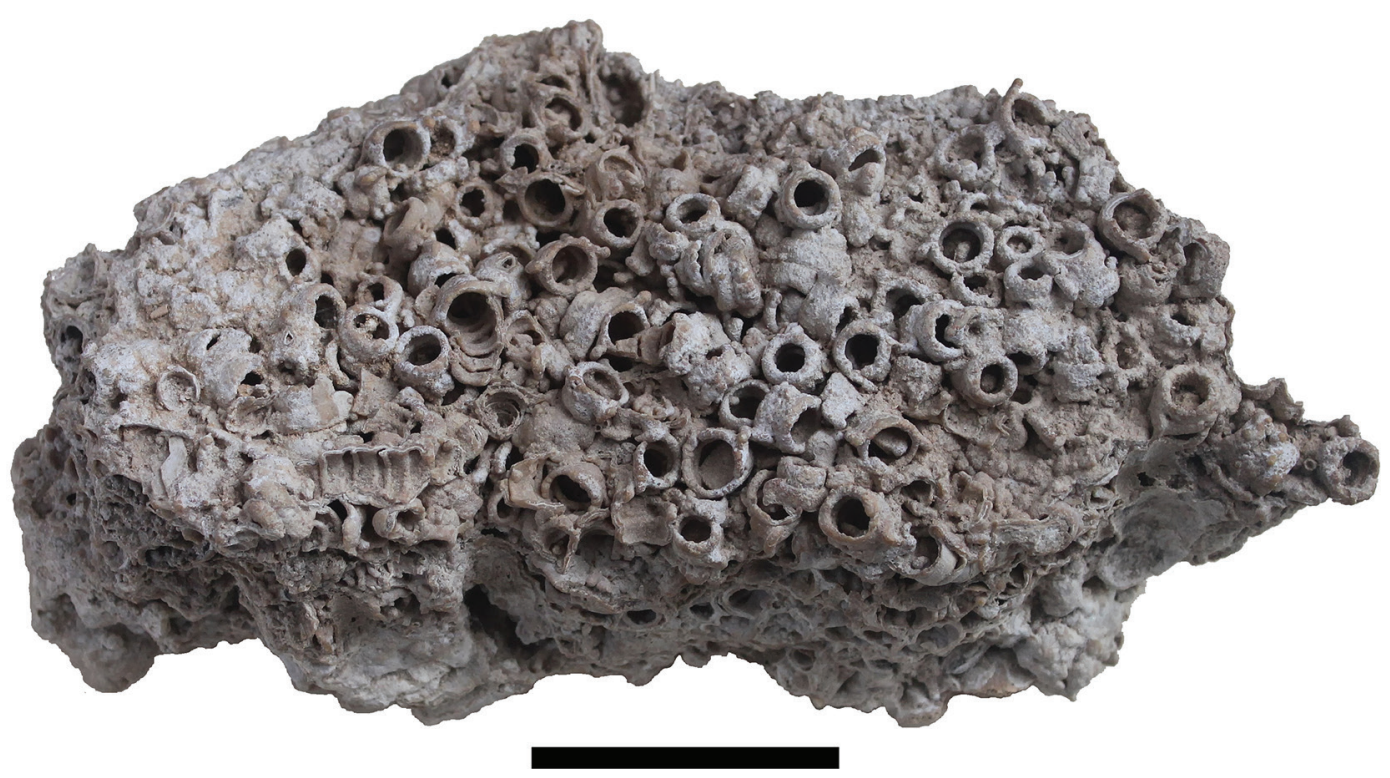

Fig. 2: Aglomerado calcáreo con restos de Diplopoda, muy probablemente Nyssodesmus sp. Escala gráfica $5 \mathrm{~cm}$.

Los diplosegmentos de estos individuos se caracterizan por poseer metazonitas con paranota o quillas horizontales y no inclinadas hacia los lados (Fig. 3C-D), ello permite descartar su pertenencia al Orden Platydesmida con el que comparte características muy similares y demuestra su pertenencia al Orden Polydesmida. Además, sus quillas son aplanadas y en algunos ejemplares se observan pequeños tubérculos en sus bordes marginales lo que permite determinar su pertenencia a la Familia Platyrhacidae (cf. Montiel, 1980).

Considerando la longitud de los diferentes diplosegmentos de la muestra de estudio y que los individuos del Orden Polydesmida están constituidos por 19 o 20 de estos diplosomitos, es posible deducir que cada ejemplar midiera entre 95 y $100 \mathrm{~mm}$ de largo; lo que corresponde al rango máximo de longitud de éste orden.

Los bordes externos de los paranota en general son lizos con ligeras proyecciones en los extremos anteriores y redondeados en su porción posterior (Fig. 3B, E, F). Sin embargo, en algunos ejemplares, como los ilustrados en la figura 3D y F, se observa que el margen externo de los paranota está dividido en dos lóbulos que se confunden con pequeñas acreciones por el carbonato de calcio que las recubre (Fig. 3D, F). Todas estas características están presentes en el género Nyssodesmus, un género muy común en Costa Rica y América Central.

Es claro que dichos segmentos corresponden a individuos diferentes y en diferentes estadios ontogenéticos, en la figura 4A, se ilustra un ejemplar de individuo juvenil donde es notable su pequeña talla. Por su parte, la numerosa acumulación de segmentos puede deberse a diversos factores, pero no necesariamente a la muda simultánea de varios individuos.

Lo que parece que ocurrió tafonómicamente fue algún tipo de selección por transporte, pues aunque son comunes los anillos aislados, son relativamente pocos los que se encuentran articulados y en ningún caso se hallaron restos más pequeños que correspondan a algún podómero, cápsula cefálica o telsón. Dicha selección probablemente se debió a escorrentía superficial dentro de la zona cárstica donde habitaban y merodeaban los diplópodos, y donde probablemente mudaban sus exoesqueletos que durante las precipitaciones eran arrastrados a alguna cavidad, desintegrándose aquellos elementos más frágiles, para finalmente depositarse en lechos sucesivos. Este tipo 


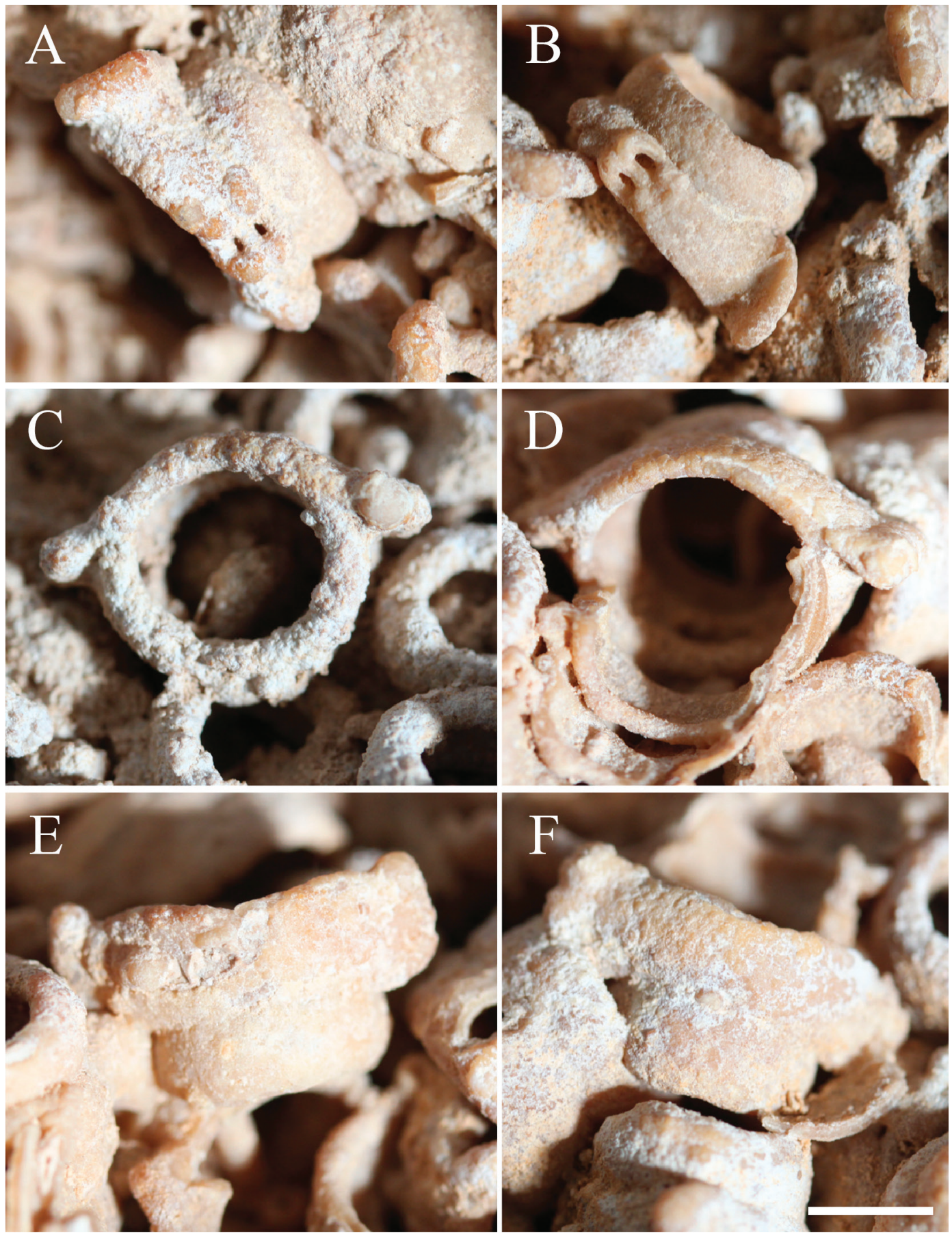

Fig. 3: Diplosomitos varios. Detalle de: A-B. las aperturas pares de la esternitas y borde anterior redondeado del paranota; C-F. paranota en posición horizontal y espinas pares recubiertas por acreciones de carbonato de calcio. Escala gráfica $5 \mathrm{~mm}$. 

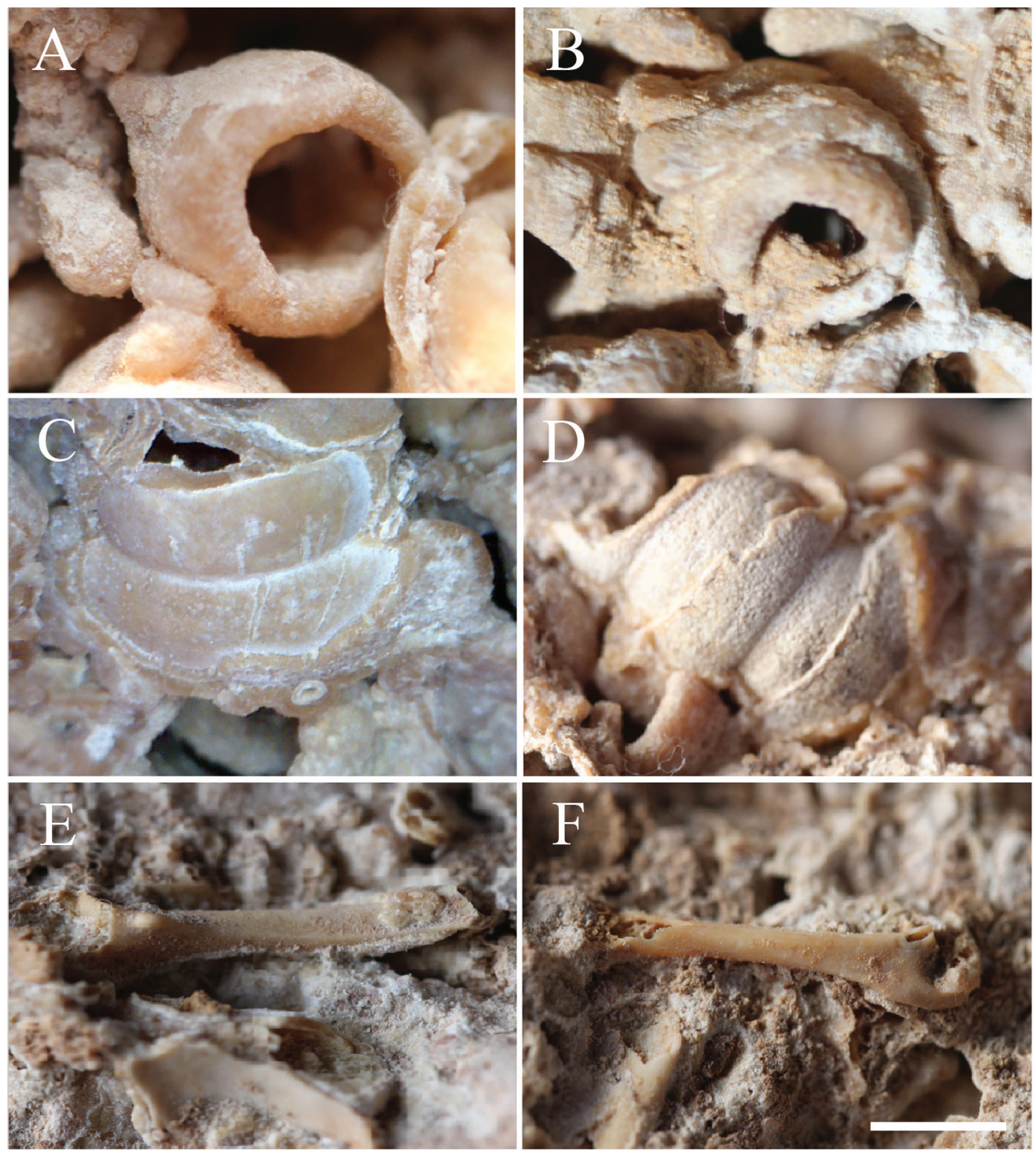

Fig. 4: A. Diplosomito de individuo juvenil; B. diplosomitos próximos a la porción caudal; C - D. detalle de la superficie dorsal y del contacto entre la prozonita y el metazonita; y huesos largos de roedores indeterminados asociados a los restos de Myriapoda: E. fragmento de ulna y F. fémur. Escala gráfica $5 \mathrm{~mm}$, excepto la figura "A" que equivale a $3 \mathrm{~mm}$.

de transporte por agua y acumulación en depresiones es la forma más común de preservación de Diplopoda, aparte de que el exoesqueleto de los Polydesmida como en otros Diplopoda está constituido por quitina reforzada con carbonato de calcio (Shear y Edgecombe, 2010), ello favoreció su cementación y preservación en el depósito de travertino que es de origen hidrotermal.

Es posible que su asociación con los roedores fuera de tipo trófico, ya que muchos roedores 
suelen depredar a los diplópodos. Además, aunque Laurito (1993) había concluido que todos los restos de roedores estaban asociados a bolos fecales y de regurgitación de aves de presa, por las características específicas y extraordinarias de su conservación, es posible que dichos restos fueran sometidos a algún tipo de transporte por el agua, misma que los condujo a la cavidad cárstica, donde se acumularon de manera conjunta con los restos de diplópodos.

\section{REFERENCIAS BIBLIOGRÁFICAS}

Almond, J. E. (1985). The Silurian-Devonian fossil record of the Myriapoda. Philosophical Transactions of the Royal Society of London, Series B, 309, 227-237.

Burger, W. (1977). Fagacea. Ann. Fieldiana Bot., $40,59-82$.

Crawford, C. S. (1992). Millipedes as model detritivores. Ber. Naturwiss. Mediz. Ver. Innsbruck, 10, 277-288.

Donovan, S. K. y Veltkamp, C. J. (1994). Unusual preservation of late Quaternary millipedes from Jamaica. Letahia, 27, 355-362.

Hoffman, R. L. (1969). Myriapoda, Exclusive of Insecta. En R. C. MOORE (ed.), Arthropoda 4. -Treatise on invertebrate paleontology, Part $R$ (vol. 2, pp. R572606). The Geological Society of America y The University of Kansas.
Hoffman, R. L., Golovatch, S. I., Adis, J. y de Morais, J. W. (2002). Diplopoda. En J. ADIS (ed.), Amazonian Arachnida and Myriapoda (pp. 505-533). Sofia: Pensoft Publisher.

Laurito, C. A. (2003). Roedores fósiles del Pleistoceno Superior de la localidad de La Palmera de San Carlos, provincia de Alajuela, Costa Rica. Revista Geológica de América Central, 29, 43-52.

Melic, A. y Grustán, D., 1996: El registro fósil de un grupo heterogéneo: Myriapoda. Boletín de la Sociedad Entomológica Aragonesa, 16, 113-124.

Montiel, L. B. (1980). Contribución a la biosistemática de la Clase Diplopoda en Costa Rica (Tesis de licenciatura inédita). Universidad de Costa Rica, San José, Costa Rica.

Pérez, E. A. (2001). Comparación de la flora fósil del Pleistoceno con la flora actual en la localidad de la Palmera de San Carlos provincia de Alajuela (Tesis de licenciatura inédita). Universidad Estatal a Distancia, San José, Costa Rica.

Pérez, E. A. (2003). Presencia de Juglans olanchana Standley y L.O. Williams (Juglandaceae) en territorio costarricense durante el Pleistoceno. Revista Geológica de América Central, 28, 77-81.

Pérez, E. A. y Laurito, C. A. (2003). Quercus corrugata Hooker (Fagacea) como indi- 
cador paleoclimático del Pleistoceno de Costa Rica. Revista Geológica de América Central, 28: 83-90.

Pérez-Asso, A. y Pérez-Gelabert, D. E. (2001). Check list of themillipeds (Diplopoda) of Hispaniola. Boletín de la Sociedad Entomológica Aragonesa, 28, 67-80.

Riquelme, F., Alvarado-Ortega, J., RamosArias, M., Hernández, M., Le Dez, I. Lee-Whiting, T. A. y Ruvalcaba-Sil, J. L. (2014a). A fossil stemmiulid millipede (Diplopoda: Stemmiulida) from the Miocene amber of Simojovel, Chiapas, Mexico. Historical Biology, 26, 415427.

Riquelme, F., Hernández-Patricio, M., MartínezDávalos, A., Rodríguez-Villafuerte, M., Montejo-Cruz, M., Alvarado-Ortega, J., Ruvalcaba-Sil, J.L. y Zuñiga-Mijangos, L. (2014b). Two flat-backed Polydesmidan millipedes from the Miocene ChiapasAmber Lagerstätte, Mexico. Plos One, 9(8), 1-8.

Santiago-Blay, J. A. y Poinar, G. O. (1992). Millipeds from Dominican amber with the description of two new species (Diplopoda: Siphonophoridae) of Siphonophora. Annals of the Entomological Society of America, 85, 363-369.

Shear, W. A. (1981). Two fossil millipeds from the Dominican amber (Diplopoda:
Chytodesmidae, Siphonophoridae). Myriapodologica, 1(8), 51-54.

Shear, W. A. y Edgecombe, G. D. (2010). The geological record and phylogeny of the Myriapoda. Arthropod Structure Development, 39, 174-190.

Shear, W. A., Selden, P. A. y Gall, J. C. (2009). Millipedes from the Grès à Voltzia, Triassic of France, with comments on Mesozoic millipedes (Diplopoda: Helminthomorpha: Eugnatha). International Journal of Myriapodology, 1, 1-13.

Shelley, R. M. (2002). A revised, annotated, family-level classification of the Diplopoda. Arthropoda Selecta, 11(3), 187-207.

Standley, P. y Williams, L. (1950). Plantas nuevas hondureñas y nicaragüenses. Ceiba, 1(2), 74-77.

Stone, D. (1977). Fagaceae: Flora Costaricensis. Fieldiana Botany, 40, 28-51.

Wilson, H. M. y Anderson, L. I. (2004). Morphology and taxonomy of Paleozoic Millipedes (Diplopoda, Chilognata: Archipolypoda) from Scotland. Journal of Paleontology, 78(1), 169-184.

Wilson, H. M., Briggs, D. E. G., Mikulic, D. G. y Klussendorf, J. (2004). Affinities of the Lower Silurian Waukesha 'myriapod'. Geological Society of America, Abstracts with Program 36, Annual Meeting, Denver, 542. 
